Article

\title{
Differential Acellular Reactive Oxygen Species (ROS) genera- tion by E-cigarettes containing Tobacco-Free Nicotine (TFN) and Tobacco-Derived Nicotine
}

\author{
Shaiesh Yogeswaran ${ }^{1}$, and Irfan Rahman ${ }^{2, *}$ \\ 1 Department of Environmental Medicine, University of Rochester Medical Center, Box 850, 601 Elmwood \\ Avenue, \\ Rochester, NY 14642, USA; Shaiesh_Yogeswaran@urmc.rochester.edu (SY); \\ *Correspondence: Irfan_Rahman@urmc.rochester.edu; Tel.: +1-(585)-275-6911
}

\begin{abstract}
Tobacco Free Nicotine (TFN) Electronic Nicotine Delivery Systems (ENDS) are yet to be classified as tobacco products; consequently, there is ambiguity over whether Food and Drug Administration (FDA) regulatory authority can be extended to include TFN e-cigarettes. In recent years, a more significant number of e-cigarette companies have been manufacturing TFN-containing e-cigarettes and e-liquids to circumvent FDA regulations. While studies have shown that aerosols generated from tobacco-derived nicotinecontaining e-cigarettes contain significant Reactive Oxygen Species (ROS) levels, no comparison studies have been conducted using TFN e-cigarettes. This study uses a single puff aerosol generator to aerosolize TFN and tobacco-derived nicotine-containing vape-products and subsequently involves semi-quantifying ROS generated by these vape products in $\mathrm{H}_{2} \mathrm{O}_{2}$ equivalents. We found that the differences between ROS levels generated from TFN and tobacco-derived nicotine-containing vape products vary by flavor. TFN tobacco flavors and fruit flavors are more toxic in terms of ROS generation than menthol/ice and drink/beverage flavored products using TFN. The type of salt nicotine used in vape products, TFN or tobacco-derived nicotine, impacts the level of ROS generated from flavored vape products. Thus, a better understanding of the type of salt nicotine used in e-cigarettes (tobacco-derived or synthetic), has modulating effect on lung oxidative stress.
\end{abstract}

Keywords: Tobacco Free Nicotine (TFN), Synthetic Nicotine, Tobacco-derived Nicotine, vape-bar, Electronic Nicotine Delivery Systems, Reactive Oxygen Species (ROS)

\section{Introduction}

Based on data from the 2021 National Youth Tobacco Survey (NYTS), a report published in the Morbidity and Mortality Weekly Report estimated 11.3\% (1.72 million) of high school students and an estimated $2.8 \%(320,000)$ of middle school students currently use e-cigarettes [1]. E-cigarette aerosols contain numerous toxic chemicals, including acrolein, formaldehyde, and acetaldehyde; the latter two are known to cause lung disease and cardiovascular disease $[2,3]$. Previous studies have shown that aerosols generated from e-cigarette vapor contain exogenous Reactive Oxygen Species (ROS) [4-6]. Additionally, studies have shown that exogenous ROS found in cigarette smoke and air pollutants can induce oxidative stress in the lungs and are the main factor in the development of Chronic $\mathrm{Ob}$ structive Pulmonary Disease (COPD) [7]. One study has shown that ROS levels within e- 
cigarette aerosols vary amongst different flavored e-cigarettes and e-cigarettes of differing nicotine concentrations [4]. Ongoing efforts to reduce youth usage of e-cigarettes include the Food and Drug Administration (FDA) extending its tobacco regulatory authority to cover Electronic Nicotine Delivery Systems (ENDS), like e-cigarettes, in 2016 [8]. More specifically, since April 2016, the FDA has required all e-cigarette manufacturers and retailers to file Premarket Tobacco Market Applications (PMTAs) to gain permission from the agency to market their products [8]. The Center for Tobacco Products (CTP) oversees all products containing tobacco-derived nicotine; however, the FDA has not decided how to regulate Tobacco-Free Nicotine (TFN) products; these products continue to remain unregulated $[2,9,10]$. In recent years, a more significant number of e-cigarette manufacturers have been using synthetic nicotine instead of tobacco-derived nicotine when producing e-cigarettes and e-liquids, all to bypass FDA regulations [9]. Synthetic nicotine is chemically identical to nicotine from tobacco plants, with the former being made within a lab without the need of a tobacco plant [11]. No studies have been conducted involving comparative analyses in exogenous ROS levels between aerosols generated by synthetic-nicotine-containing e-cigarettes and those by e-cigarettes containing tobacco-derived nicotine. With the substantial rise in youth usage of e-cigarettes and a more significant number of e-cigarette manufacturers producing TFN e-cigarettes, more studies examining differences in ROS levels between aerosols generated by tobaccobased nicotine and synthetic nicotine-containing e-cigarettes are needed [10]. In this study, we quantify ROS levels generated by synthetic nicotine-containing ENDS products and compare them to ROS levels generated from their flavor-specific tobacco-derived nicotine-containing counterparts.

\section{Materials and Methods}

\subsection{Procurement of Vape-bars and E-liquids}

Three different TFN vape-bars and three different TFN e-liquids were analyzed in this study (Table 1). In addition to the six TFN vape-products analyzed, six different tobacco-derived nicotine-containing vape-bars were analyzed in this study. All vape-products (vape-bars and e-liquids) used in this study were either purchased from online vendors or local stores in the Rochester, NY area. 
Table 1. Tobacco-derived and tobacco-free nicotine ENDS used in this study

\begin{tabular}{|c|c|c|c|}
\hline Company & Flavor & $\begin{array}{c}\text { Nicotine Concentration } \\
\text { (\%) }\end{array}$ & Nicotine Salt-Type \\
\hline Air Factory & Pink Punch (Pink Punch Lemonade) & $5.00 \%$ & TFN \\
\hline Bad Drip & Rawberry Melon & $5.00 \%$ & TFN \\
\hline Flair Plus & Pink Lemonade & $5.00 \%$ & Tobacco-Derived \\
\hline Glas (BASIX Series) & Blue Razz & $5.00 \%$ & TFN \\
\hline Hyppe & Blue Raz & $5.00 \%$ & Tobacco-Derived \\
\hline Hyde & Spearmint & $5.00 \%$ & Tobacco-Derived \\
\hline JUUL & Virginia Tobacco & $5.00 \%$ & Tobacco-Derived \\
\hline Lit & Strawmelon & $5.00 \%$ & TFN \\
\hline Pachamama & Banana Ice & $5.00 \%$ & Tobacco-Derived \\
\hline Puff Bar & Banana Ice & $5.00 \%$ & TFN \\
\hline Salty Man & Creamy Tobacco & $5.00 \%$ & TFN \\
\hline Salty Man & Spearmint & $5.00 \%$ & \\
\hline
\end{tabular}

\subsection{Acellular ROS Quantification within Generated Aerosols}

ROS levels within aerosols generated from all twelve vape-products were quantified via spectrofluorometry and in $\mathrm{H}_{2} \mathrm{O}_{2}$ equivalents. Aerosols from each individual TFN vape-product used in the study were generated using a Buxco Individual Cigarette Puff Generator (Data Sciences International (DSI), St. Paul, MN, USA) (Cat\#601-2055-001) (Figure 1). Once aerosols were generated from the individual vape-bar, the aerosols were exposed to $10 \mathrm{~mL}$ of fluorogenic dye at $1.5 \mathrm{~L} / \mathrm{min}$; this continued for one puffing regiment (Figure 1). One puffing regiment lasted for 10 minutes; 2 puffs $/ \mathrm{min}$, with each puff having a volume of $55.0 \mathrm{~mL}$. The fluorogenic dye used in the study was made from $0.01 \mathrm{~N} \mathrm{NaOH}, 2^{\prime} 7^{\prime}$ dichlorofluorescein diacetate ( $\left.\mathrm{H}_{2} \mathrm{DCF}-\mathrm{DA}\right)$ (EMD Biosciences, San Diego, CA, USA) (Cat \# 287810), phosphate ( $\left.\mathrm{PO}_{4}\right)$ buffer, and horseradish peroxidase (Thermo Fisher Scientific, Waltham, MA, USA (Cat\# 31491). Each TFN e-liquid was aerosolized using a new, empty refillable JUUL Pod (OVNStech, Shenzen, GD, China) (Mo: WO1 JUUL Pods) inserted into a JUUL device (JUUL Labs Inc., Washington, DC, USA) (Mo: Rechargeable JUUL Device w/USB charger). Subsequently, this JUUL device was inserted into the Individual Cigarette Puff Generator.

Each vape-bar and JUUL Pod containing TFN e-liquid had undergone three separate puffing regiments to prepare three individual samples of $10 \mathrm{~mL}$ dye solution exposed to e-cigarette aerosols. For our negative control, filtered air was passed through fluorogenic dye using the previously mentioned puffing regiment and inserting a filter into the Individual Puff Generator instead of an e-cigarette. For our positive control, the smoke generated from a conventional cigarette (Kentucky Tobacco Research \& Development Center in the University of Kentucky, Lexington, KY, USA) (Mo: 3R4F) was exposed to fluorogenic dye under the previously mentioned puffing regiment.

Subsequently, $0 \mu \mathrm{M}, 10 \mu \mathrm{M}, 15 \mu \mathrm{M}, 20 \mu \mathrm{M}, 30 \mu \mathrm{M}, 40 \mu \mathrm{M}$, and $50 \mu \mathrm{M} \mathrm{H}_{2} \mathrm{O}_{2}$ standards were prepared using $30 \% \mathrm{H}_{2} \mathrm{O}_{2}$ (Thermo Fischer Scientific, Waltham, MA, USA) (Cat\# H323-500) and double-distilled water $\left(\mathrm{ddH}_{2} \mathrm{O}\right)$. After aerosolizing each vape product and exposing its generated aerosols to three separate $10 \mathrm{~mL}$ samples of fluorogenic dye, each resulting fluorogenic dye sample and standard was placed in a $37^{\circ} \mathrm{C}$ degree water bath (VWR 1228 Digital Water Bath) for fifteen minutes. After placing each sample and standard into the water bath, the resulting solutions were analyzed via fluorescence spectroscopy ( $E x=475 \mathrm{~nm}$ and $E m=535 \mathrm{~nm}$ ). Readings were taken on a spectrofluorometer (Turner Quantech fluorometer, Mo. FM109535) in fluorescence intensity units (FIU) and measured as $\mathrm{H}_{2} \mathrm{O}_{2}$ equivalents. 


\subsection{Statistical Analyses}

One-way ANOVA and Tukey's post-hoc test for multiple pairwise comparisons via GraphPad Prism Software version 8.1.1 was used to conduct statistical analyses of significance. Samples were run in triplicates. The results are shown as mean $\pm \mathrm{SEM}$ with triplicate analyses. Data were considered to be statistically significant for $p$ values $<0.05$.

\section{Results}

3.1. Differences in ROS Levels within aerosols generated by TFN vape-products and TobaccoDerived Nicotine-containing vape-products vary with flavor

For the blueberry-raspberry-flavored vape-products analyzed, the level of ROS generated from the Hyppe: Blue Raz (5.0\% tobacco-derived nicotine) bar ( 4.92-6.61 $\mu \mathrm{M}$ ) did not significantly differ from that generated from the GLAS Basix Blue Razz (5.0\% synthetic nicotine) e-liquid (4.97-7.44 $\mu \mathrm{M})$ [Figure 1a]. Among the strawberry watermelon flavored vape-bars analyzed, the difference in Acellular ROS levels in aerosols generated by the Bad Drip: Rawberry Melon (5.0\% synthetic nicotine) vape-bar (3.82-7.48 $\mu \mathrm{M})$ and Lit: Strawmelon (5.0\% tobacco-derived nicotine) vape-bar (4.10-4.77 $\mu \mathrm{M})$ was not significant [Figure 1b].

Regarding minty/iced (cooled) flavored vape products, there appear to be significant differences in ROS levels generated between TFN vape products and their corresponding flavor-specific tobacco-derived nicotine counterparts [Figure 2]. The level of ROS generated from the Pachamama: Banana Ice (5.0\% synthetic nicotine) vape-bar (7.19-8.40 $\mu \mathrm{M})$ differed significantly from that generated from the Puff Bar: Banana Ice (5.0\% tobacco-derived nicotine) bar $(9.69-15.87 \mu \mathrm{M})$ [Figure 2a]. Similarly, the level of ROS generated from aerosolized Salty Mann: Spearmint (5.0\% synthetic nicotine) eliquid (1.33-2.11 $\mu \mathrm{M})$ differed significantly from that generated from the Hyde: Spearmint (5.0\% tobacco-derived nicotine) bar (3.28-4.50 $\mu \mathrm{M})$ [Figure $2 \mathrm{~b}$ ].

When comparing tobacco-flavored vape products, the level of ROS generated from the aerosolized Salty Man: Creamy Tobacco (5.0\% synthetic nicotine) e-liquid (2.32-3.96 $\mu \mathrm{M})$ did not significantly differ from that generated from the JUUL: Virginia Tobacco $(5.0 \%$ tobacco-derived nicotine) bar (1.26-5.14 $\mu \mathrm{M})$ [Figure 3a]. However, regarding drinkflavored ENDS, the level of ROS generated from the Flair Plus: Pink Lemonade (5.0\% tobacco-derived nicotine) bar $(1.84-2.47 \mu \mathrm{M})$ was significantly different from that generated from the aerosolized Air Factory: Pink Punch (5.0\% synthetic nicotine) eliquid (0.61-0.92 $\mu \mathrm{M})$ [Figure $3 \mathrm{~b}]$. 


\section{Figures}

\section{Tobacco-Derived Nicotine vs. Synthetic Nicotine (Blueberry-Raspberry)}
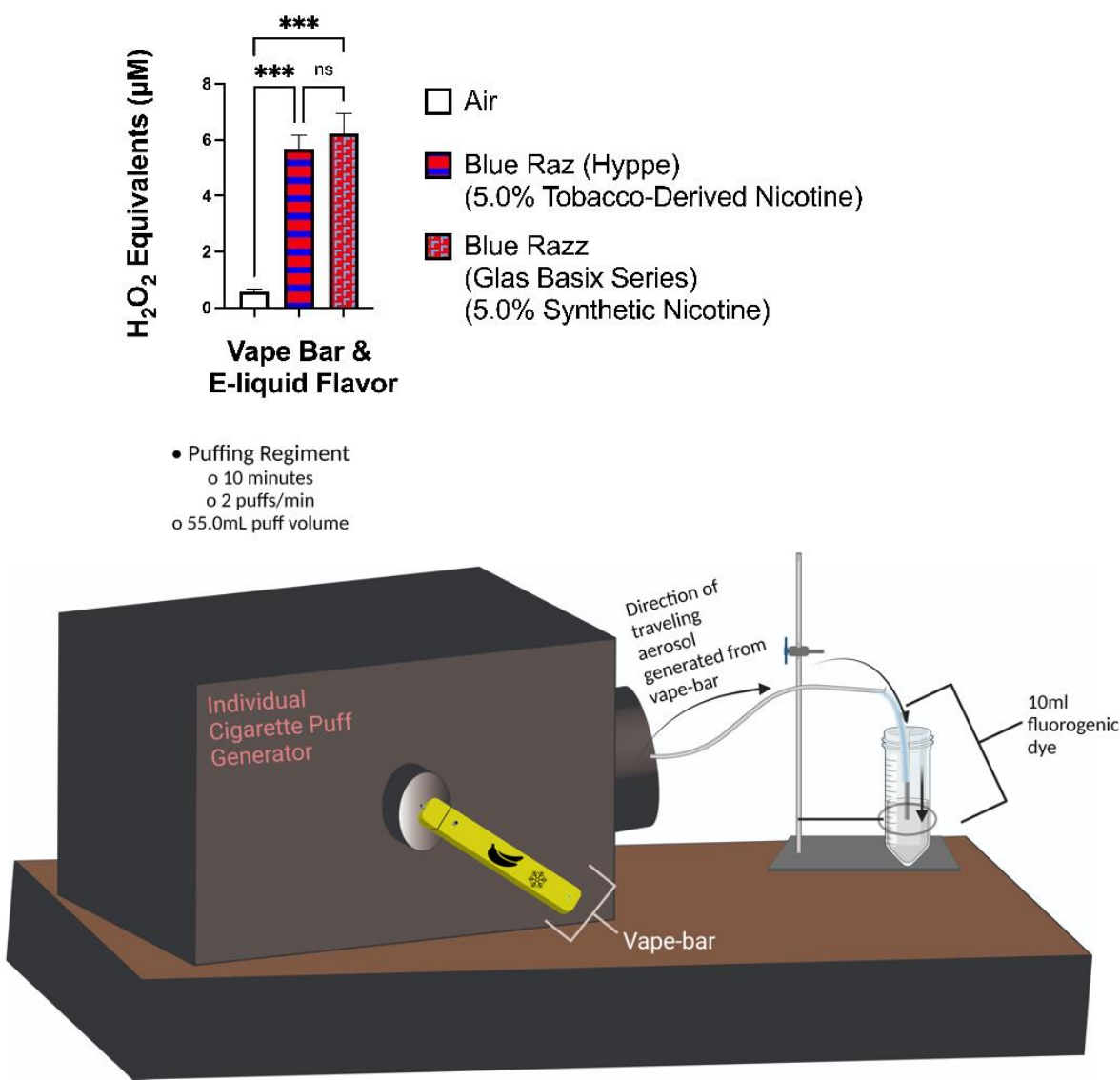

Figure 1: E-cigarette puff generator apparatus. The schematic shows the apparatus used to aerosolize each vape-bar and e-liquid included in this study. Once inserted into the Individual Cigarette Puff Generator, the component e-liquid within each vape bar was aerosolized for one individual puffing regiment; the generated aerosol was then exposed to $10 \mathrm{~mL}$ of fluorogenic dye during those ten minutes. One puffing regiment consisted of a vape-bar being aerosolized for 10 minutes and generating 20 total puffs; each puff lasting 3.0 seconds and having a volume of $55.0 \mathrm{~mL}$. The entirety of the aerosolization process and the subsequent exposure of the generated aerosols to fluorogenic dye was done within a chemical fume hood. The pictogram was made using Adobe Illustrator and BioRender. 
(a)

Tobacco-Derived Nicotine vs. Synthetic Nicotine (Strawberry Melon)

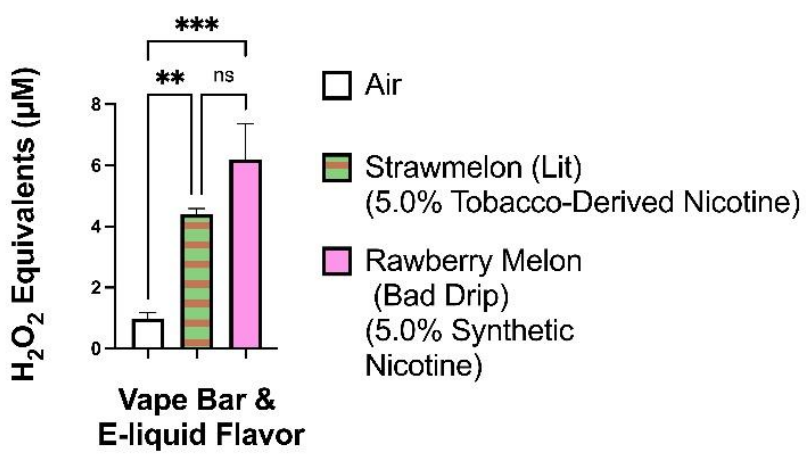

(b)

Figure 2: ROS levels within aerosols generated from fruity-flavored tobacco-derived nicotinecontaining and TFN vape-products ROS levels within the generated aerosols from each individual TFN vape-product and tobacco-derived nicotine-containing vape-product was measured via spectrofluorometry and quantified as $\mathrm{H}_{2} \mathrm{O}_{2}$ equivalents. During analysis, the level of ROS generated from each individual vape-product was compared to the ROS generated from the filtered air control. Data are represented as mean \pm SEM, and significance was determined by one-way ANOVA. ${ }^{*} \mathrm{p}<$ $0.05,{ }^{* *} \mathrm{p}<0.01,{ }^{* * *} \mathrm{p}<0.001$, and ${ }^{* * * *} p<0.0001$ versus air controls. NS is abbreviated for "NonSignificant" versus air-controls $(p>0.05)$. NS is abbreviated for "Non-Significant" versus air-controls $(p>0.05)$. Sample size $(\mathrm{N})=3-4$ 


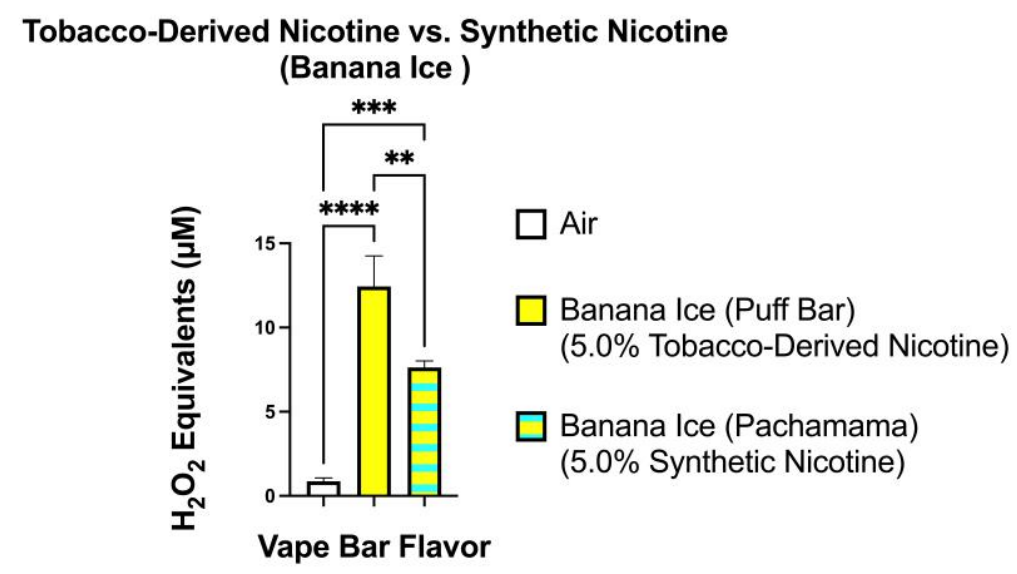

(a)

Tobacco-Derived Nicotine vs. Synthetic Nicotine (Spearmint)

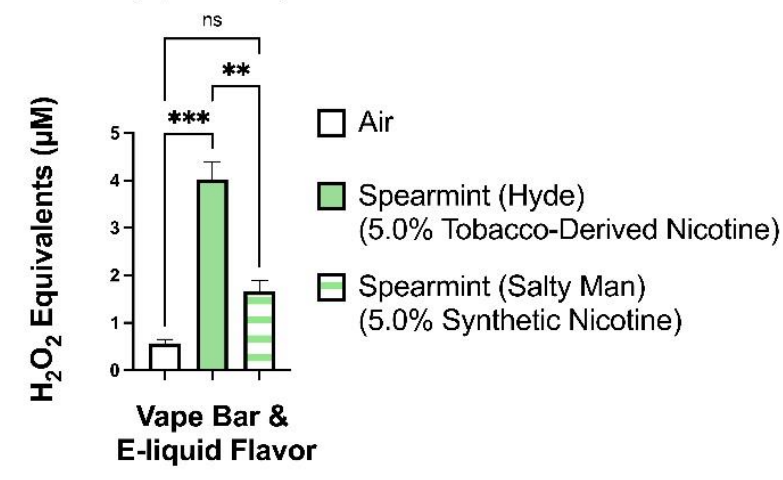

(b)

Figure 3: ROS generation among aerosols generated from minty/iced (cooled) flavored TFN and tobacco-derived nicotine-containing vape-products ROS levels within the generated aerosols from each individual minty/iced (cooled) flavored TFN and tobacco-derived nicotine-containing vapeproduct was measured via spectrofluorometry and quantified as $\mathrm{H}_{2} \mathrm{O}_{2}$ equivalents. During analysis, the level of ROS generated from each individual vape-bar was compared to the ROS generated from the filtered air control. Data are represented as mean \pm SEM, and significance was determined by one-way ANOVA. ${ }^{*} \mathrm{p}<0.05,{ }^{* *} \mathrm{p}<0.01$, and ${ }^{* *} \mathrm{p}<0.001$ versus air controls. NS is abbreviated for "Non-Significant" versus air-controls $(p>0.05)$. NS is abbreviated for "Non-Significant" versus aircontrols $(p>0.05)$. Sample size $(\mathrm{N})=3-4$ 


\section{Tobacco-Derived Nicotine vs. Synthetic Nicotine} (Tobacco)

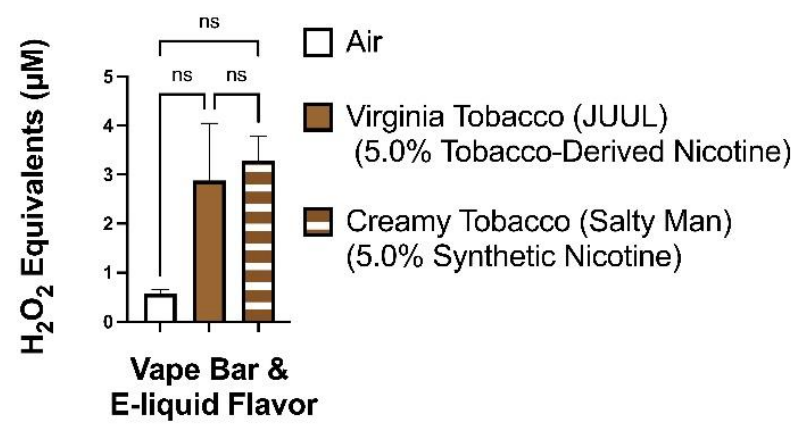

(a)

\section{Tobacco-Derived Nicotine vs. Synthetic Nicotine} (Pink Lemonade)

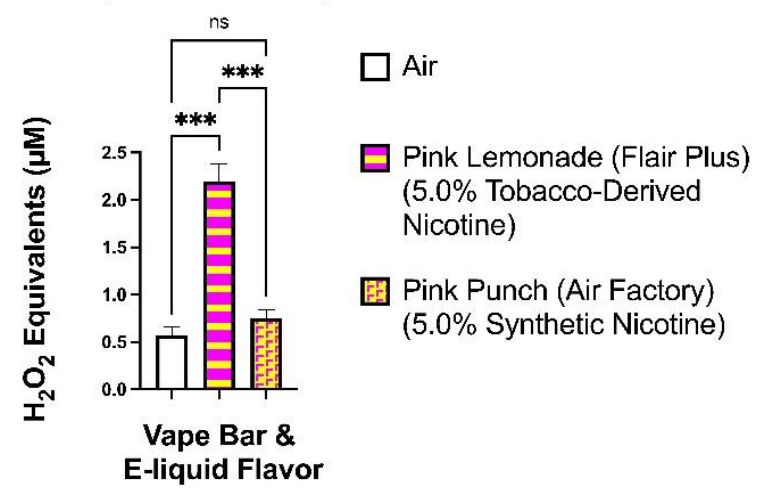

(b)

Figure 4: ROS generation among aerosols generated from tobacco and drink flavored TFN and tobacco-derived nicotine-containing vape-products ROS levels within the generated aerosols from each individual tobacco and drink-flavored TFN e-liquid and tobacco-derived nicotine-containing vape-bar was measured via spectrofluorometry and quantified as $\mathrm{H}_{2} \mathrm{O}_{2}$ equivalents. During analysis, the level of ROS generated from each individual vape-bar was compared to the ROS generated from the filtered air control. Data are represented as mean $\pm S E M$, and significance was determined by one-way ANOVA. ${ }^{*} \mathrm{p}<0.05,{ }^{* *} \mathrm{p}<0.01$, and ${ }^{* * *} \mathrm{p}<0.001$ versus air controls. NS is abbreviated for "Non-Significant" versus air-controls $(p>0.05)$. NS is abbreviated for "Non-Significant" versus aircontrols $(p>0.05)$. Sample size $(\mathrm{N})=3-4$

\section{Discussion}

Our data suggest that the type of nicotine salt used in e-liquids and vape-bars, tobacco-derived or synthetic, plays a role in modulating ROS generation upon component e-liquid aerosolization. To further explain, significant differences in ROS generation were observed between TFN and tobacco-derived nicotine-containing vape-products containing drink and minty/iced flavoring. However, non-significant differences in ROS generation were observed between TFN and tobacco-derived nicotine-containing vape-products with fruity and tobacco flavoring. Not only does our data suggest that the type of nicotine salt used in ENDS products plays a role in modulating exogenous ROS generation, but also that flavoring agents used in e-cigarettes play a role in modulating ROS generated from vape products.

Similarly, the results of our study seem to concur with our previous study, the data of which suggested that flavoring agents used in tobacco-derived nicotine-containing 
vape-bars play a role in modulating ROS generation upon component e-liquid aerosolization [4]. Regarding the effects of nicotine content on ROS generation and oxidative stress, one study had found that nicotine increases oxidative stress in rat mesencephalic cells in a dose-dependent manner [12]. Another study found that aerosols from flavored e-cigarettes and e-liquids promoted oxidative stress in H292 lung epithelial cells as well as in the lungs of mice [13]. Additionally, one study found that ROS generated from e-cigarettes was highly dependent on the flavor of e-liquid used (fruity and tobacco) [5]. However, studies examining the differences in ROS generation within cellular and acellular systems due to the usage of tobacco-derived nicotine-containing and TFN vape- products are lacking. While previous studies have shown that voltage, flavoring, and nicotine concentration have a role in modulating e-cigarette generated ROS levels, the results of our study show that the type of nicotine salt used (synthetic or tobacco-derived) does as well [4-6].

Interestingly, we noticed that amongst the minty/cooled flavored vape-products analyzed (Spearmint and Banana Ice), the level of ROS generated by the synthetic-nicotine vape-product was significantly less than that generated by its flavor specific tobacco-derived nicotine-containing counterpart. Additionally, amongst the drink-flavored vapeproducts analyzed, the synthetic nicotine-containing vape product generated significantly less ROS than its tobacco-derived nicotine-containing counterpart. Synthetic nicotine lacks the impurities contained within tobacco-derived nicotine. Similarly, the differences in exogenous ROS between aerosols generated by TFN and tobacco-derived nicotine-containing vape-products with Pink Lemonade, Spearmint, and Banana-Ice flavoring may be due to the differences in impurities within each type of nicotine salt (tobacco-derived or synthetic) used. However, to determine whether the results observed for the Pink Punch Lemonade, Spearmint, and Banana Ice flavored ENDS are due to differences in the level of impurities within the salt nicotine used, e-cigarette screening via Inductively coupled plasma mass spectrometry (ICP-MS) is needed.

Regarding the limitations of this study, due to there being very few companies that manufacture both TFN and tobacco-derived nicotine-containing vape-products, we could not control for the e-cigarette brand in our pairwise comparisons between TFN products and their flavor specific tobacco-derived nicotine-containing counterparts. Certain vendors do not manufacture tobacco flavors TFN because of non-regulation of tobacco flavors in tobacco-derived nicotine vape products due to lack of public interests. One study has shown that even amongst e-cigarettes of the same flavor, ROS levels within generated aerosols vary by brand [4]. Future studies examining the differences in ROS levels generated by TFN vape products and their flavor-specific tobacco-derived nicotine-containing counterparts of the same company are needed.

\section{Conclusions}

Our data suggest that TFN tobacco flavors and fruit flavors are more toxic in terms of ROS generation than menthol/ice and drink/beverage flavored products using TFN . In other words, beverage flavor and minty/iced (cool) flavored TFN products generate significantly less ROS than their corresponding flavor-specific tobacco-derived nicotinecontaining counterparts. Our study provides a better understanding of the role the type of salt nicotine used in e-cigarettes, tobacco-derived or synthetic, has in modulating e-cigarette-induced oxidative stress in the lungs.

\section{Patents}

None.

\section{Supplementary Materials: None}

Author Contributions: “Conceptualization, IR.; methodology, IR and SY.; software, S, Y.; validation, SY, IR formal analysis, SY; investigation, SY.; resources, IR data curation, SY writing - original draft 
preparation, SY; writing - review and editing,; IR, editing,; SY and IR visualization, SY.; supervision, IR.; project administration, IR.; funding acquisition, IR All authors have read and agreed to the published version of the manuscript.

Funding: This research was supported by our TCORS Grant: CRoFT 1 U54 CA228110-01.

Institutional Review Board Statement: All assays and experiments performed in this study were approved and in accordance with the University of Rochester Institutional Biosafety Committee. Additionally all protocols, procedures, and data analysis in this study followed the NIH guidelines and standards of reproducibility and scientific rigor by an unbiased approach. (Biosafety Study approval \#Rahman/102054/09-167/07-186; identification code: 07-186; date of approval: 01/05/2019). No animals or human subjects were used.

Informed Consent Statement: Not applicable as study did not involve humans.

Data Availability Statement: We declare that we have provided all the data in figures.

Acknowledgments: Figure 1 was made using BioRender and Adobe Illustrator. Figures 2 and 3 were made using GraphPadprism.

Conflicts of Interest: The authors declare no conflict of interest.

\section{References}

1. Park-Lee, E., et al., Notes from the Field: E-Cigarette Use Among Middle and High School Students - National Youth Tobacco Survey, United States, 2021. MMWR Morb Mortal Wkly Rep, 2021. 70(39): p. 1387-1389.

2. $\quad$ Bein, K. and G.D. Leikauf, Acrolein - a pulmonary hazard. Mol Nutr Food Res, 2011. 55(9): p. $1342-60$.

3. Ogunwale, M.A., et al., Aldehyde Detection in Electronic Cigarette Aerosols. ACS Omega, 2017. 2(3): p. $1207-1214$.

4. Yogeswaran, S., T. Muthumalage, and I. Rahman, Comparative Reactive Oxygen Species (ROS) Content among Various Flavored Disposable Vape Bars, including Cool (Iced) Flavored Bars. Toxics, 2021. 9(10).

5. Zhao, J., et al., Assessment of reactive oxygen species generated by electronic cigarettes using acellular and cellular approaches. J Hazard Mater, 2018. 344: p. 549-557.

6. Haddad, C., et al., Reactive Oxygen Species Emissions from Supra-and Sub-Ohm Electronic Cigarettes. J Anal Toxicol, 2019. 43(1): p. $45-50$.

7. Rahman, I. and W. MacNee, Role of oxidants/antioxidants in smoking-induced lung diseases. Free Radic Biol Med, 1996. 21(5): p. 669-81.

8. Food and H.H.S. Drug Administration, Deeming Tobacco Products To Be Subject to the Federal Food, Drug, and Cosmetic Act, as Amended by the Family Smoking Prevention and Tobacco Control Act; Restrictions on the Sale and Distribution of Tobacco Products and Required Warning Statements for Tobacco Products. Final rule. Fed Regist, 2016. 81(90): p. 28973-9106.

9. Cwalina, S.N., et al., Tobacco-free Nicotine - New Name, Same Scheme? N Engl J Med, 2021. 385(26): p. $2406-2408$.

10. Jordt, S.E., Synthetic nicotine has arrived. Tob Control, 2021.

11. Zettler, P.J., N. Hemmerich, and M.L. Berman, Closing the Regulatory Gap for Synthetic Nicotine Products. Boston Coll Law Rev, 2018. 59(6): p. 1933-1982.

12. Barr, J., et al., Nicotine induces oxidative stress and activates nuclear transcription factor kappa B in rat mesencephalic cells. Mol Cell Biochem, 2007. 297(1-2): p. 93-9.

13. Lerner, C.A., et al., Vapors produced by electronic cigarettes and e-juices with flavorings induce toxicity, oxidative stress, and inflammatory response in lung epithelial cells and in mouse lung. PLoS One, 2015. 10(2): p. e0116732. 